\title{
Exploration of susceptible genes associated with Henoch-Schönlein purpura by whole exome sequencing
}

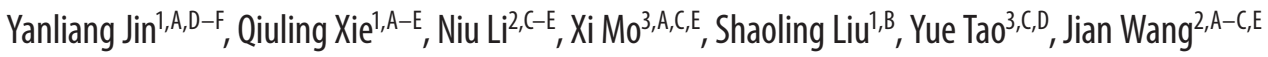 \\ ${ }^{1}$ Department of Rheumatology, Shanghai Children's Medical Center, Shanghai Jiaotong University School of Medicine, China \\ ${ }^{2}$ Department of Medical Genetics, Institute of Pediatric Translational Medicine, Shanghai Children's Medical Center, Shanghai Jiaotong University School of Medicine, China \\ ${ }^{3}$ Institute of Pediatric Translational Medicine, Shanghai Children's Medical Center, Shanghai Jiaotong University School of Medicine, China \\ A - research concept and design; B - collection and/or assembly of data; C - data analysis and interpretation; \\ $D$ - writing the article; $E$ - critical revision of the article; $F$ - final approval of the article
}

Advances in Clinical and Experimental Medicine, ISSN 1899-5276 (print), ISSN 2451-2680 (online)

Adv Clin Exp Med. 2019;28(9):1199-1207

\section{Address for correspondence}

Yanliang Jin

E-mail: jinyanliang2000@163.com

Funding sources

Fund of Science and Technology Development and Innovation of Pudong New Area, Shanghai, China (No. PKJ2012-Y51).

Conflict of interest

None declared

\section{Acknowledgements}

We want to express our thanks to all the patients who participated in this study and to all the people who have considerably contributed to the paper preparation.

Received on January 25, 2018

Reviewed on March 2, 2018

Accepted on February 7, 2019

Published online on August 19, 2019

\begin{abstract}
Background. Henoch-Schönlein purpura (HSP) is a systemic small-vessel vasculitis caused by environmental and inherent factors. Although recent research has advanced our understanding of the role of genetic susceptibility in HSP, there are still significant gaps in our knowledge.

Objectives. In this study, we aimed to explore some susceptibility genes likely associated with HSP.

Material and methods. Three DNA samples from a family with HSP were used to perform whole exome sequencing with Illumina Hiseq 2500 high-throughput sequencing. The relevant single nucleotide variants (SNVS) were screened according to specific filter conditions and the screened SNVs were then verified with Sanger sequencing. The Sanger sequencing results were further screened according to available literature. Finally, candidate genes were validated in 92 samples from children with HSP, and also in 1 child with HSP from HSP family, using the polymerase chain reaction technique (PCR).

Results. Our analysis revealed that the MIF gene and the MGAT5 gene related to immunity remained after screening. Among the 93 children with HSP, there were 3 patients with MIF mutations and 2 patients with MGAT5 mutations.

Conclusions. Our findings are helpful for providing new methods and ideas for understanding the pathogenesis of HSP by detecting and analyzing gene mutations at the whole-exome level including multi-generation sequencing. MIF and MGATS may be new susceptibility loci for HSP, but their roles in the pathogenesis of HSP are worthy of further study.
\end{abstract}

Key words: Henoch-Schönlein purpura, whole exome sequencing, MIF, MGAT5

Cite as

Jin $Y, X i e Q$, Li N, et al. Exploration of susceptible genes associated with Henoch-Schönlein purpura by whole exome sequencing. Adv Clin Exp Med. 2019;28(9):1199-1207. doi:10.17219/acem/103800

DOI

10.17219/acem/103800

Copyright

Copyright by Author(s)

This is an article distributed under the terms of the Creative Commons Attribution Non-Commercial License (http://creativecommons.org/licenses/by-nc-nd/4.0/) 


\section{Introduction}

Henoch-Schönlein purpura (HSP) is a small-vessel vasculitis that often occurs in children and can cause various clinical issues, such as skin purpura, arthritis, and/or arthralgia, abdominal pain, and renal involvement. ${ }^{1}$ Some of the renal damage can progress to renal insufficiency, which determines the long-term prognosis of HSP. ${ }^{2}$ Although the first HSP report was made nearly 200 years ago, the exact etiology and pathogenesis of the disease are still unclear.

Some studies have shown that the incidence of HSP has a tendency for familial aggregation. ${ }^{3-5}$ In other words, genetic factors may play an important role in the pathogenesis of HSP. The past few years have seen a tremendously large amount of research in quest for genetic variants which may influence HSP susceptibility. Candidate gene investigations have since revealed roles in HSP susceptibility of an increasing number of genes, including TGF- $\beta$, MEFV (E148Q), RAS, P-selectin, C1GALT1, HSP70-2, TNF- $\alpha, I L-17$ (rs2275913), CTLA-4, PAF-AH, eNOS, iNOS, $T L R-4, A C E$, etc., all of which have been validated in independent Chinese populations. ${ }^{6-17}$ The above studies, however, were basically designed to study the correlation between specific genes and HSP, but these selected genes are not necessarily related to the incidence of hereditary HSP.

Besides, the occurrence of HSP is related to immune disorders, such as a Th1/Th2 imbalance and overactive Th2 cells. ${ }^{18}$ Therefore, we set out to perform whole exome sequencing (WES) in a family with HSP to find genes related to immune abnormalities which are likely associated with HSP, so as to provide clues for researching the pathogenesis of HSP.

\section{Material and methods}

\section{Patients}

The study focused on a Chinese family, consisting of a boy with HSP, his father (who has had HSP since he was 10 years old) and the boy's mother, who does not suffer from HSP. Other 92 samples from unrelated patients with HSP were recruited from the Department of Rheumatism at Shanghai Children's Medical Center. Henoch-Schönlein purpura was diagnosed according to criteria established by the European League Against Rheumatism (EULAR) in $2010 .{ }^{19}$

The 12-year-old boy (sample No. WES-741) presented with HSP on both of his lower limbs 2 days prior; this was accompanied by joint pain, but no renal involvement. His father (sample No. WES-742) had suffered from HSP since the age of 10 , accompanied by abdominal pain and gastrointestinal bleeding, though his mother (sample No. WES-743) had no history of HSP.
The study was approved by the Medical Ethics Committee of Shanghai Children's Medical Center, Shanghai Jiaotong University School of Medicine, China, and informed consent was obtained from the parents of all participants.

\section{Whole exome sequencing}

The genomic DNA of the 3 samples was isolated from $2 \mathrm{~mL}$ of peripheral blood samples collected from the veins using a QIAamp Blood DNA Mini Kit ${ }^{\circledR}$ (Qiagen GmbH, Hilden, Germany). A total of $3 \mu$ g of DNA from the sample was processed through shearing using a Covarias ${ }^{\circledR}$ M220 Ultrasonicator system (Covaris Inc., Woburn, USA) resulting in an amplicon of $150-200 \mathrm{bp}$. The genomic DNA library was prepared with an Agilent SureSelect Target Enrichment System (Agilent Technologies, Inc., Santa Clara, USA) according to the manufacturer's instructions. Both coding exons and the flanking intronic regions were enriched with SureSelect XT Human All Exon V6 (Agilent Technologies). Clusters were then generated with isothermal bridge amplification using an Illumina cBot station, and sequencing was performed on an Illumina HiSeq 2500 System (Illumina Inc., San Diego, USA).

Base calling and sequence read quality assessment were performed using Illumina Sequence Control Software with Real Time Analysis software (Illumina). The sequence reads to a reference human genome (Human 37.3, SNP135) were aligned using NextGENe (SoftGenetics, State College, USA). All single-nucleotide variants (SNVs) and indels were saved in VCF format and uploaded to Ingenuity Variant Analysis (Ingenuity Systems, Redwood City, USA) for biological analysis and interpretation.

\section{Single nucleotide variant screening}

The screening parameters of the candidate variants used with the Ingenuity software consisted of 5 parts: 1) exclusion of low confidence variants; 2) allele frequency under $1.0 \%$ in the 1000 Genomes Project, $1.0 \%$ of the NHLBI ESP exomes, $1.0 \%$ of the Allele Frequency Community, or $1.0 \%$ of the ExAC database, with an area of analysis including each exon and about 20 bp of exon-intron boundaries; 3) exclusion of benign variants, including synonymous, harmless missense predicted by PolyPhen- 2 and SIFT software, when those predicted had no impact on splicing using MaxEntScan software; 4) a filtering index of clinical symptoms of immunity for analysis of the resulting candidate variants; and 5) taking the intersecting set of variants in the cases of WES-741 and WES-742.

\section{Sanger sequencing verification}

The primers for the amplicon of the screened SNVs gained by University of California, Santa Cruz (UCSC Genome Browser Home) were designed using Primer3 online 
software (http://genome.ucsc.edu/cgi-bin/hgGateway) and synthesized with Map Biotechnology (Shanghai, China). The amplicon length was 300-500 bp and the annealing temperature was set at $60^{\circ} \mathrm{C}$.

The exons and the exon-intron boundaries were amplified using polymerase chain reaction (PCR). The PCR products $(5 \mu \mathrm{L})$ were examined on a $1 \%$ agarose gel and purified using an ExoSAP-IT Kit (GE Healthcare, Cleveland, USA). Sequencing reactions were prepared with the BigDye ${ }^{\circledR}$ Direct Cycle Sequencing Kit (Thermo Fisher, Waltham, USA). The final products were purified from agarose gel using QIAquick Gel Extraction Kit (Qiagen) and the capillary electrophoresis sequencing was performed by using an ABI Prism 3730XL Genetic Analyzer (Thermo Fisher) with the forward and reverse primers. The sequence data were analyzed using Mutation Surveyor ${ }^{\circledR}$ software v. 4.0.4 (SoftGenetics).

\section{PCR of MIF and MGAT5 and analysis of data}

All exons of MIF and MGAT5 were amplified using PCR from the genomic DNA of 93 children with HSP. The primers for the amplicons of MIF (GenBank accession No. NM_002415) and MGAT5 (GenBank accession No. NM_002410) were designed using UCSC ExonPrimer online software (http://genome.ucsc.edu/index.html) and were synthesized using Map Biotechnology (Shanghai, China). The primers designed for MIF and MGAT5 are listed in Table 1 (A and B). The exons and the exon-intron boundaries were amplified using PCR. The reaction mixture for each amplicon of $M I F$ contained $1 \times$ Premix Taq (Ex Taq v. 2.0; cat. No. RR 003; Takara/Biotechnology, Co. Ltd., Kusatsu, Japan), 100 ng of genomic DNA, and $1 \mu \mathrm{L}$ of forward and reverse primer in a final volume of $25 \mu \mathrm{L}$. The reaction was performed under the following PCR conditions: initial denaturation at $95^{\circ} \mathrm{C}$ for $5 \mathrm{~min}$; then 19 cycles of $95^{\circ} \mathrm{C}$ for $30 \mathrm{~s}, 65^{\circ} \mathrm{C}$ for $30 \mathrm{~s}$ and $72^{\circ} \mathrm{C}$ for $45 \mathrm{~s} ; 14$ cycles of $95^{\circ} \mathrm{C}$ for $30 \mathrm{~s}, 55^{\circ} \mathrm{C}$ for $30 \mathrm{~s}$ and $72^{\circ} \mathrm{C}$ for $45 \mathrm{~s}$; and a final elongation step at $72^{\circ} \mathrm{C}$ for $5 \mathrm{~min}$ using a C1000 ${ }^{\mathrm{TM}}$ Thermal Cycler PCR instrument (Bio-Rad Laboratories Inc., Hercules, USA). The PCR products were examined on a $1 \%$ agarose gel (Sangon Biotechnology Co. Ltd., Shanghai, China) with electrophoresis and purified using a QIAquick Gel Extraction kit (Qiagen). The purified DNA was sequenced using an ABI3730XL sequencer (Thermo Fisher) with forward and reverse primers. The sequence data was analyzed using Mutation Surveyor DNA Variant Analysis v. 4.0.4 (SoftGenetics). The same reaction system and conditions were used for the amplification of MGAT5.

After PCR analysis, we used Bioedit (V.7.1.3) (Tom Hall, http://www.mbio.ncsu.edu/BioEdit/bioedit.html) to find pathogenic predictions of all variants of MIF and MGAT5
Table 1A. The PCR primer sequence for the MIF gene

\begin{tabular}{|l|c|}
\multicolumn{1}{|c|}{ Primer name } & Primer sequence (5'-3') \\
\hline MIF-E1F & CAGGCGGTGACTTCCCCA \\
\hline MIF-E1R & CCCCGAACGTCCACTTCG \\
\hline MIF-E2-3F & AGGGGACAGGAAGAGGGG \\
\hline MIF-E2-3R & GACCTCGCTCCCCAATATCC \\
\hline
\end{tabular}

Table 1B. The PCR primer sequence for the MGAT5 gene

\begin{tabular}{|c|c|}
\hline Primer name & Primer sequence $\left(5^{\prime}-3^{\prime}\right)$ \\
\hline MGAT5-E1F & AGTGAAAACATGGCTTCTGGT \\
\hline MGAT5-E1R & GGCTCTCTGTGTGCAATTCA \\
\hline MGAT5-E2F & CGGCCCTTGTCATCTTTCAG \\
\hline MGAT5-E2R & ACTTATCTTTACAATGGCCATGC \\
\hline MGAT5-E3F & TATATTGGAGCTGGAGGGGC \\
\hline MGAT5-E3R & CATAGCCACAGACAAAGCCG \\
\hline MGAT5-E4F & TGCACACCTGGCTATCTTGA \\
\hline MGAT5-E4R & GGGAGAGTAATGAAGGACGGA \\
\hline MGAT5-E5F & TCATCTGAGAGGGCACTTGT \\
\hline MGAT5-E5R & TCCACAGAGACGAATACAGAAGT \\
\hline MGAT5-E6F & CAGTTTGCAATCCTGGGACC \\
\hline MGAT5-E6R & AGACTGAGCAGATTCACCCT \\
\hline MGAT5-E7F & CCAAGGGCTGCAGTGATTTT \\
\hline MGAT5-E7R & ATGAAGCCTCTGGAGCCTTC \\
\hline MGAT5-E8F & TTTGAAAGGGCCATGCTGTC \\
\hline MGAT5-E8R & GCAACACTCCATACAGCTGA \\
\hline MGAT5-E9F & TCTTGTCTGGTCCTGCTTCT \\
\hline MGAT5-E9R & ACAGCACACACTTCTACAGC \\
\hline MGAT5-E10F & ACTGGGAATGTGTGGGAGAG \\
\hline MGAT5-E10R & GCAGGTACATACAGCCTTGG \\
\hline MGAT5-E11F & TCCACAAAGTCCAGCCAGAT \\
\hline MGAT5-E11R & GCTGCCATTGGTCTAGCAAA \\
\hline MGAT5-E12F & AGCCTTCCAAAGTGAGGTGT \\
\hline MGAT5-E13R & AGCCTCTATACCCTCACCCT \\
\hline MGAT5-E13F & TCAAGGGGAAGGACACAGTG \\
\hline MGAT5-E13R & CCAAATCCTGCAGCTTGGTT \\
\hline MGAT5-E14F & GCTTGAGATCACGACTTGCA \\
\hline MGAT5-E14R & GGTGACCATGACAGTGCTAC \\
\hline MGAT5-E15F & CTCCCAACTCTTCCCCGTC \\
\hline MGAT5-E15R & CTTATCCCCACAGCCCAGTA \\
\hline MGAT5-E16F & GATTGCAGACGAAGAGGGTG \\
\hline MGAT5-E16R & GCAATGCCAATTCAGAACCTTC \\
\hline
\end{tabular}

and analyzed their conservation; the properties of amino acids were found using the Hphob./Kyte \& Doolittle scale (http://web.expasy.org/protscale/). 


\section{Results}

\section{DNA sequencing}

The 3 samples from the HSP family were screened for selected variants using WES. The sequencing quality parameters are listed in Table 2. After being screened for selected variants, the filtered SNVs were verified using Sanger sequencing; the results are listed in Table 3. Functional prediction and biological relevance analysis led to 2 genes: MIF and MGAT5. One variant was in MGAT5 (c.673C>G; p.L225V) and the other variant was in $M I F$ (c.295T>C; p.Y99H). The 2 variants were both missense mutations which were heterozygous in both the patient and his father (Fig. 1). The pathogenicity analysis of all variants of MGAT5 and MIF are listed in Table 4.

\section{PCR analysis of the MGAT5 gene in 93 children with HSP}

The sequencing results of $M G A T 5$ showed that there were 3 synonymous variants (c.141C $>\mathrm{T}, \mathrm{c} .267 \mathrm{~A}>\mathrm{G}$, and c.348A $>\mathrm{G}$ ) and 2 missense variants (c.673C $>\mathrm{G}$ and c. $1117 \mathrm{~A}>\mathrm{G}$ ). The 3 synonymous variants do not cause changes in the corresponding amino acid and every synonymous variant exists in only 1 HSP patient. The other 2 missense variants are both heterozygous mutations and are located in the encoding region of MGAT5. Base mutation can cause amino acid change (c.1117A > G, p.Met373Val; c.C673G, p.Leu225Val). Also, each missense variant exists in only 1 HSP patient.

Conservation analysis of MGAT5 amino acid sequences in different species was performed with Bioedit software (Fig. 2). The results showed that the above amino acid sites
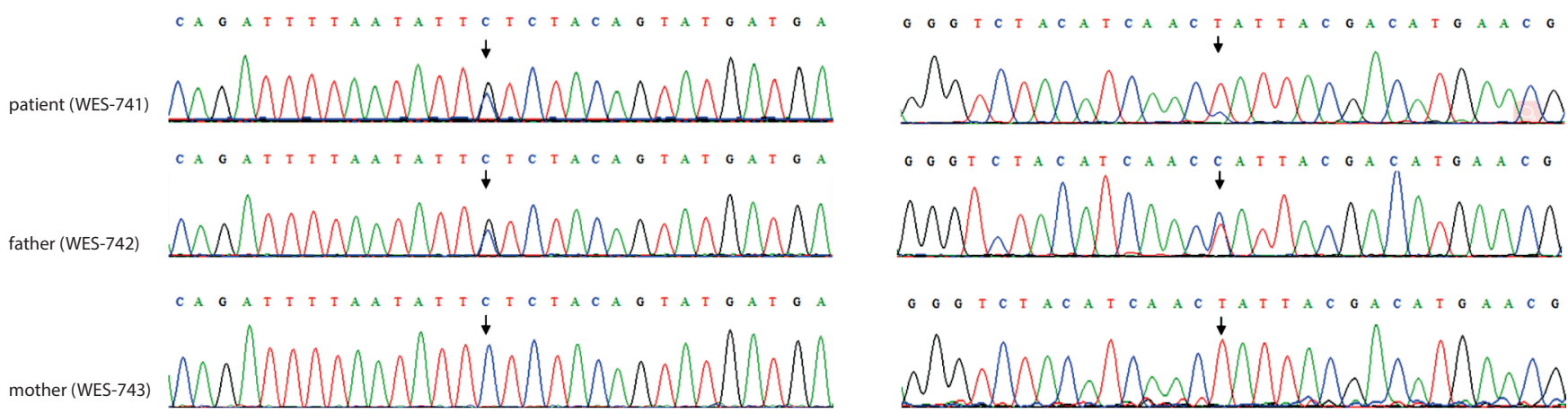

Fig. 1. Sanger sequencing of MGAT5 and MIF genes. The MGAT5 c. 673C>G; p.L225V and MIF c.295T>C; p.Y99H variants were detected in the patient and his father. The patient's mother had no variants. The corresponding variant bases are indicated by arrows

$\begin{array}{ll}\text { H. sapiens } & \text { LHSIGKIGGA QNRSYSKLLC GLLAERLRIS PDRVYINYYD MNAANVGWNN STFA } \\ \text { P.troglodytes } & \text { LHSIGKIGGA QNRPHSKLLC GLLAERLRIS PDRVYINYYD MNAANVGWNN STFA } \\ \text { S. scrofa } & \text { LHSIGKIGGA QNRSYSKLLC GLLAERLRIS PDRIYINYYD MNAANVGWNG STFA } \\ \text { B.taurus } & \text { LHSIGKIGGA QNRSYSKLLC GLLTERLRIS PDRIYINFCD MNAANVGWNG STFA } \\ \text { R. norvegicus } & \text { LHSIGKIGGA QNRNYSKLLC GLLSDRLHIS PDRVYINYYD MNAANVGWNG STFA } \\ \text { M. musculus } & \text { LHSIGKIGGA QNRNYSKLLC GLLSDRLHIS PDRVYINYYD MNAANVGWNG STFA } \\ \text { G.gallus } & \text { LYSIGKIGGQ QNKTYTKLLC DMIAKHLHVS ADRVYINFD INAANVGWNG STFA } \\ \text { T.sirtalis } & \text { LHSIGKISAP QNKAYSAILS ALLAKRLHVP ADRVYINFYD MAPANVGWNG STFA } \\ \text { X. laevis } & \text { LCSIGKIGGP QNKSYTKLLC DILTKQLNIP ANRVYINYYD LNAANVGWNG STFA } \\ \text { D. rerio } & \text { LTSIGKISGA QNKQYSKLLM GLLNKHLGVS ADRIYINFVD MDPANVAWNN STFG } \\ \text { P.falciparum } & \text { ITSIGGINRS NNSALADQIT KLLVSNLNVK SRRIYVEFRD CSAQNFAFSG SLFG }\end{array}$

Fig. 2. Conservation analysis of mutations in MIF. One variant (c.295T>C, p.Y99H; in yellow) is well-conserved in vertebrates except B. taurus and D. rerio. Another variant (c.338C >T, p.T1131; in green) is well-conserved across species

Table 2. The sequencing quality parameters of the 3 cases included in this study

\begin{tabular}{|c|c|c|c|c|c|c|}
\hline Sample & Total reads & $\begin{array}{c}\text { Average read } \\
\text { depth }(x)\end{array}$ & $\begin{array}{l}\text { Percent of ROI } \\
\text { with } 1 \times[\%]\end{array}$ & $\begin{array}{l}\text { Percent of ROI } \\
\text { with } 20 \times[\%]\end{array}$ & $\begin{array}{l}\text { Unaligned } \\
\text { reads }\end{array}$ & $\begin{array}{l}\text { Off-target percent } \\
\text { of reads [\%] }\end{array}$ \\
\hline Patient (WES-741) & 59938725 & 101.95 & 96.897 & 99.873 & 2625970 & 28.88 \\
\hline Father (WES-742) & 69050718 & 115.8 & 97.658 & 99.884 & 3382470 & 29.353 \\
\hline Mother (WES-743) & 57841691 & 101.44 & 97.044 & 99.776 & 2191901 & 27.118 \\
\hline
\end{tabular}

$\mathrm{NA}$ - not available; $\mathrm{ROI}$ - region of interest. 


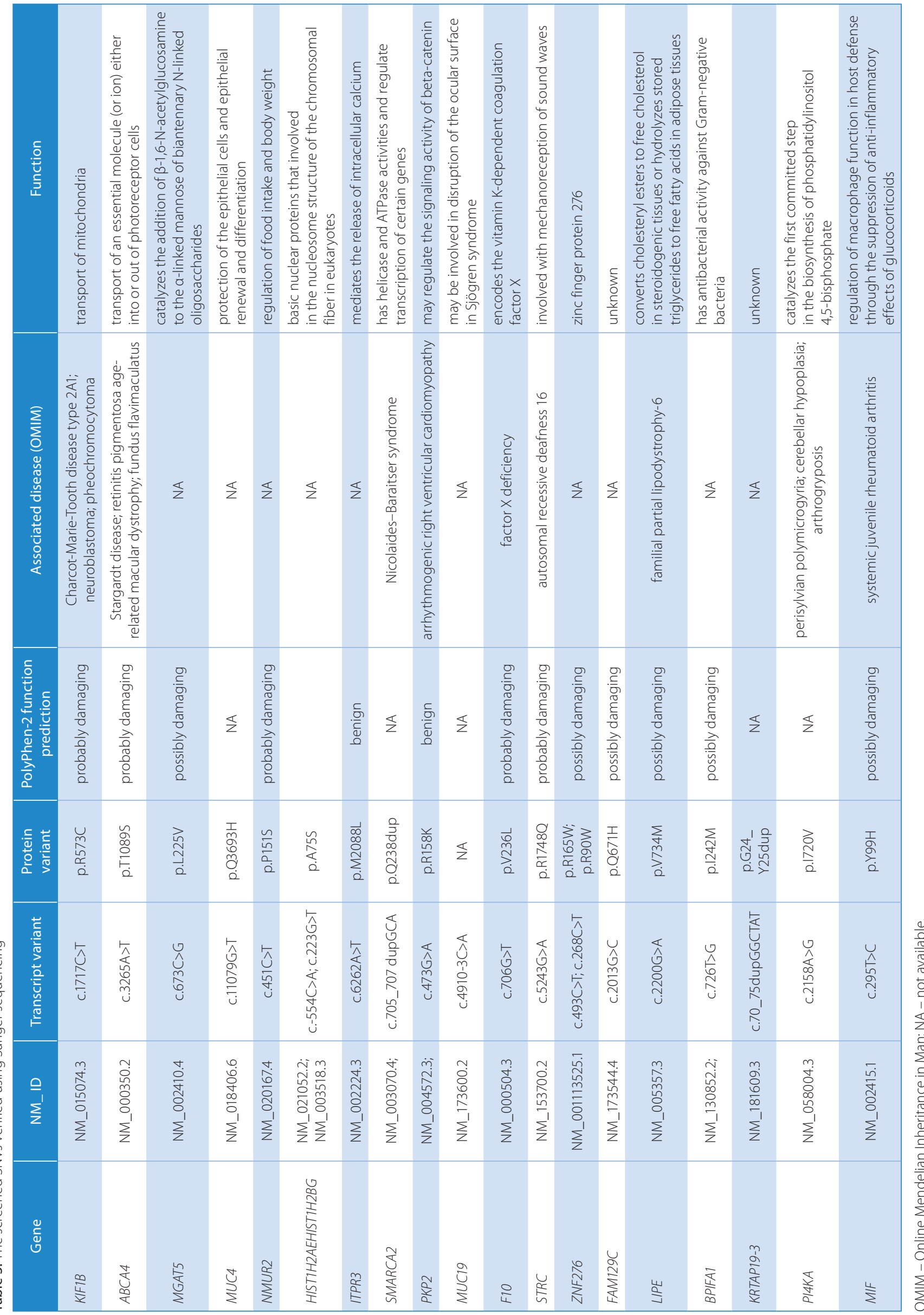




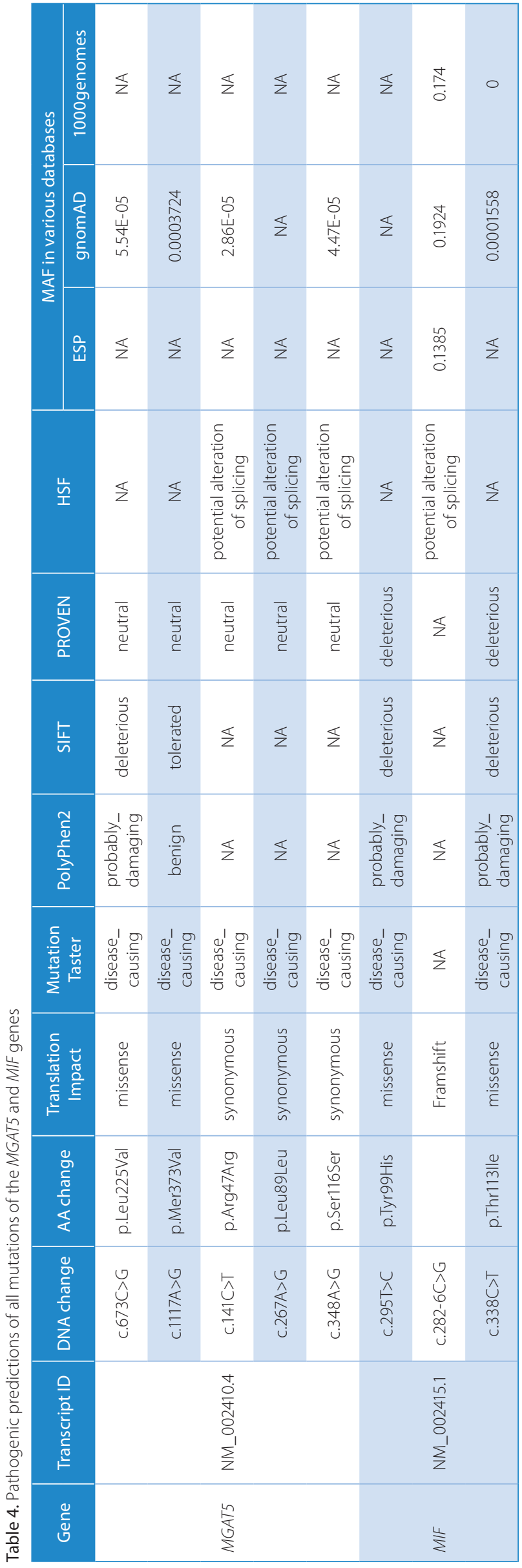

were highly conserved among different species. This suggests that the mutation of the 2 conserved sites may be important factors affecting the functioning of MGAT5, so the mutations are not caused by natural selection in evolution.

According to the Hphob./Kyte \& Doolittle scale, the individual values for methionine, valine and leucine are 1.9, 4.2 and 3.8, respectively. These amino acids are hydrophobic and their properties do not change significantly. Because of the 2 highly conserved sites, the 2 gene mutations may still cause structural and functional changes of the MGAT5 protein.

\section{PCR analysis of the MIF gene in 93 children with HSP}

The sequencing results of $M I F$ showed that the 3 variants, c.282-6C>G, c.295T >C and c.338C >T, were all heterozygous mutations. The c.282-6C>G variant (rs2070766) is located in the splicing region of MIF. Among the 93 children, 35 patients (37.6\%) carried this mutation. These data are a close match to those of the European population (32.2\%) and the East Asian population (28.6\%) according to the ExAC database, and the allele frequency is greater than $3 \%$, so these sites are the single nucleotide polymorphism (SNP) site.

The c. $295 \mathrm{~T}>\mathrm{C}$ and c.338C $>\mathrm{T}$ variants are both located in the encoding region of MIF. Base mutation can result in a change of the corresponding amino acid (c.338C $>\mathrm{T}$; p.Thr113Ile and c.295T>C; p.Tyr99His). Among the 93 patients with HSP, there were $2(2.15 \%)$ c.338C $>\mathrm{T}$ variants and $1(1.08 \%)$ c. $295 \mathrm{~T}>\mathrm{C}$ variant.

Conservation analysis of MIF amino acid sequences in different species were also performed with Bioedit software (Fig. 3). The results showed that the $113^{\text {th }}$ amino acid was highly conserved among different species. This suggests that the variant may be one of the factors that affect the functioning of $M I F$, rather than natural selection in evolution. And the $99^{\text {th }}$ amino acid is conserved in other vertebrates except cattle and zebrafish, in which it is semi-conserved.

Using the Hphob./Kyte \& Doolittle scale, the individual values for tyrosine, histidine, threonine, and leucine are $-1.3,-3.2,-0.7$, and 4.5 , respectively. The p.Tyr99His variant leads to a significant change in the structure of the amino acid side chain, while p.Thr113Ile can cause its hydrophobicity to change greatly. The amino acid side chain structure changes as well. Therefore, the 2 amino acids change more significantly.

\section{Discussion}

Henoch-Schönlein purpura is one of the most common types of vasculitis in children, but its cause is still unknown and, so far, no specific genetic abnormality has been described in patients with HSP. ${ }^{20-22}$ In this study, a WES analysis was performed in a family with HSP. Considering 


$\begin{array}{lll}\text { H. sapiens } & \text { IRTDFNILYS MMKHEEFRW } & \text { H. sapiens } \\ \text { P. troglodytes } & \text { IRTDFNILYS MMKHEEFRW } & \text { P. troglodytes } \\ \text { C. familiaris } & \text { IRTDFNILYS MMKHEEFRW } & \text { C. familiaris } \\ \text { B. taurus } & \text { IRTDVNILYS MKKHEEFRW } & \text { B. taurus } \\ \text { R. norvegicus } & \text { IRTDFNILYG MMKHEEFRW } & \text { R. norvegicus } \\ \text { M. musculus } & \text { IRTDFNILYG MMKHEEFRW } & \text { M. musculus } \\ \text { G. gallus } & \text { IRINFDPLYK MSRHEEFRW } & \text { G. gallus } \\ \text { T. sirtalis } & \text { IRTNFDLLYK MSRHEEFRW } & \text { T. sirtalis } \\ \text { X. tropicalis } & \text { IRTDFDDLYK MAKHEEFRW } & \text { X.tropicalis } \\ \text { D. rerio } & \text { VRTSFDELYR MSRREEFRW } & \text { D. rerio }\end{array}$

QFKKTLGPSW VHYQCMLRVL DSFGTEPEFN
QFKKTLGPSW VHYQCMLRVL DSFGTEPEFN
QFKKTLGPSW VHYQCMLRVL DSFGTEPEFN
QFKKTLGPSW VHYQCMLRIL DSFGTEPEFN
QFKKTLGPSW VHYQCMLRVL DSFGTEPEFN
QFKKTLGPSW VHYQCMLRVL DSFGTEPEFN
QFKKTLGPSW VHYQCMLRVL DSFGTEPEFN
QFKKTLGPSW VHYQMLRVL DSFGTEPEFN
QFKKTLGPSW VHYCMLRVL DSFGTEPEFN
QFKKTLGPSW VHYQCMLRVL DSFGTEPEFN

Fig. 3. Conserved analysis of mutations in MGAT5. The 2 variants (c.673C>G, p.L225V; left, in yellow, and c.1117A>G, p.M373V; right, in green) are both highly conserved across species

that HSP is associated with immune imbalance, we gained a total of 20 SNVs in the 20 different genes screened. Among the screened markers for immunity in our whole exome association study, MGAT5 and MIF were of particular interest by functional prediction and biological relevance analysis.

The MGAT5 gene, also known as GNT-V, encodes beta1,6 N-acetyl-glucosaminyltransferase, an enzyme which catalyzes the addition of beta-1,6 GlcNAc- to N-glycans on glycoproteins. An MGAT5 deficiency increases the number of $\mathrm{T}$-cell receptors recruited to the antigenpresenting surface, thereby reducing the need for CD28 co-receptor engagement. MGAT5 functions as a negative regulator of T-cell activation thresholds and susceptibility to immune diseases, such as multiple sclerosis or rheumatoid arthritis. ${ }^{23-25}$ The expression of beta-1,6 GlcNAcbranched N-linked glycans selectively inhibits Th1 cell differentiation and enhances the polarization of Th2 cells. ${ }^{26}$ In our study, PCR analysis of MGAT5 in the 93 HSP patients showed that there was 1 patient (1.08\%) with the c. $1117 \mathrm{~A}>\mathrm{G}$ variant and another 1 child $(1.08 \%)$ with the c. $673 \mathrm{C}>\mathrm{G}$ variant. Our result for the $\mathrm{c} .673 \mathrm{C}>\mathrm{G}$ variant $(1.08 \%)$ is much higher than that of the East Asian population $(0.83 \%)$ and the European population (0\%). The frequency of the c.1117A $>$ G variant in HSP patients (1.08\%) is also far higher than that of the East Asian population $(0.43 \%)$ and the European population (0\%). According to the analysis of MATG5 gene sequence conservation and amino acid properties, these 2 amino acids did not change significantly, but because the 2 sites are highly conserved their mutation may still lead to structural and functional changes of the MGAT5 protein. In addition, the HSP patient with the c.673C $>\mathrm{G}$ variant, whose father also had HSP in childhood, had joint pain at his initial diagnosis. Once he had received enough prednisone therapy, his rash was under control and his joint pain was relieved. The patient with c. $1117 \mathrm{~A}>\mathrm{G}$ had a rash which lasted 2 months, with a longer course and kidney involvement, and he recovered after 1 year of prednisone treatment. We speculate that the mutation of the MGAT5 gene may be related to the occurrence of HSP, severity of HSP and the duration of glucocorticoid treatment through some unknown mechanism. Of course, more research is needed on the reliability of these findings and on the intrinsic mechanism of the disease.

Another interesting marker in our screening is macrophage migration inhibitory factor (MIF). It is a pro-inflammatory cytokine that is produced by immune T cells, macrophages and monocytes, and plays pivotal roles in innate and acquired immunity. ${ }^{27}$ It has been reported to inhibit random migration and concentration of macrophages at sites of inflammation and to induce pro-inflammatory cytokine secretion, leading to an excessive inflammatory response. ${ }^{28,29}$ The functional effects of $M I F$ gene polymorphisms have been identified and associated with susceptibility or severity of several acute, chronic and autoimmune inflammatory conditions and vasculitides. ${ }^{30}$ The promoter region of the MIF -173G/C polymorphism has been identified as a functionally relevant variant of this gene. ${ }^{31}$ Our previous study and other studies have found increased levels of MIF in the urine and renal tissues of children with HSP. ${ }^{32,33}$ However, Nalbantoglu et al. and Amoli et al. showed that the MIF gene $-173 \mathrm{G} / \mathrm{C}$ polymorphism was not associated with HSP. ${ }^{34,35}$ The above results indicate that the $-173 \mathrm{G} / \mathrm{C}$ polymorphism in the MIF gene promoter does not appear to be a genetic risk factor for HSP, and that HSP may be related to abnormal regulation of inflammation by the MIF protein, abnormal levels of which are caused by other mutations in MIF. Our study demonstrated 2 new variants in the MIF gene. One is the c.295T>C variant; it was found in 1 patient (1.08\%) with HSP and the site had never been reported before. Therefore, the gene frequency cannot be compared with the normal population of the ExAC database. The other variant is the c.338C $>\mathrm{T}$ variant. There were 2 children (2.15\%) carrying this variant, and its frequency is much higher than that of the populations of East Asia (0.28\%) and Europe (0\%). According to the analysis of MIF sequence conservation and amino acid properties, the $99^{\text {th }}$ amino acid is semi-conserved, the $113^{\text {rd }}$ is highly conserved and the properties of the 2 amino acids change significantly. Therefore, the 2 variants 
are likely to lead to changes in the structure and function of MIF, which is related with HSP.

Additionally, glucocorticoid (GC), which has a broad spectrum of anti-inflammatory and immune-regulatory effects on host immune responses, can inhibit the activation of immune cells and reduce the production of pro-inflammatory mediators, including cytokines, prostaglandins and reactive oxygen species. ${ }^{36}$ Because of these properties, $\mathrm{GC}$ is effective in the treatment of some inflammatory diseases. ${ }^{37}$ As a unique trans-regulator of GC, MIF can antagonize endogenous and exogenous glucocorticoid activity, so as to maintain a balance in immune regulation. ${ }^{38}$ However, the research on the systemic lupus erythematosus (SLE) showed that MIF was associated not only with disease severity, ${ }^{39}$ but also with steroid resistance. ${ }^{40}$ In our study, there were 2 children with HSP and the c.338C $>\mathrm{T}$ variant. One was diagnosed with HSP nephritis, which was refractory and lasted for 2 years. The proteinuria was relieved after prednisone treatment combined with immunosuppressant. The other was a girl with severe rash (both lower limbs and both upper limbs) at her initial diagnosis who was treated with prednisone. The patient with the c.295T $>C$ variant had joint pain at his initial diagnosis, and his rash was controlled after sufficient prednisone treatment. Thus, we speculate that MIF may be related to the severity of HSP. The specific mechanism between $M I F$ and the occurrence of HSP, the relationship between $M I F$ and the severity of the disease, and the drug resistance of the hormone still need to be studied further.

Because the sample size in this study is small, it needs to be verified in larger samples. At the same time, it also needs to be further studied in gene function and animal models. So far, few studies have reported a relationship between MGAT5 or MIF and the pathogenesis of HSP, but our study provides a new direction for future research.

\section{References}

1. Chimenti MS, Ballanti E, Triggianese P, Perricone R. Vasculitides and the complement system: A comprehensive review. Clin Rev Allergy Immunol. 2015;49(3):333-346.

2. Davin JC, Coppo R. Henoch-Schönlein purpura nephritis in children. Nat Rev Nephrol. 2014;10(10):563-573.

3. Kiryluk K, Moldoveanu Z, Sanders JT, et al. Aberrant glycosylation of $\lg A 1$ is inherited in both pediatric IgA nephropathy and HenochSchönlein purpura nephritis. Kidney Int. 2011;80(1):79-87.

4. Zhang Y, Gu W, Mao J. Sibling cases of Henoch-Schönlein purpura in two families and review of literature. Pediatr Dermatol. 2008;25(3): 393-395.

5. Chen YH, Lin TY, Chen CJ, Chen LK, Jan RH. Familial cases of HenochSchönlein purpura in Taiwanese Aborigines. Pediatr Neonatol. 2012; 53(5):320-324

6. Yang YH, Lai HJ, Kao CK, Lin YT, Chiang BL. The association between transforming growth factor-beta gene promoter C-509T polymorphism and Chinese children with Henoch-Schönlein purpura. Pediatr Nephrol. 2004;19(9):972-975.

7. He X, Lu H, Kang S, et al. MEFV E148Q polymorphism is associated with Henoch-Schönlein purpura in Chinese children. Pediatr Nephrol. 2010;25(10):2077-2082.

8. Desong L, Fang L, Songhui $Z$, et al. Renin-angiotensin system gene polymorphisms in children with Henoch-Schönlein purpura in West China. J Renin Angiotensin Aldosterone Syst. 2010;11(4):248-255.
9. Li J, Wang YB, Liu HL, et al. P-selectin gene -2123 polymorphism in children with Henoch-Schönlein purpura [in Chinese]. Zhongguo Dang Dai Er Ke Za Zhi. 2011;13(4):278-281.

10. An J, Lu Q, Zhao H, Cao Y, Yan B, Ma Z. A study on the association between C1GALT1 polymorphisms and the risk of Henoch-Schönlein purpura in a Chinese population. Rheumatol Int. 2013;33(10):2539-2542.

11. Ding GX, Wang CH, Che RC, et al. Heat shock protein 70-2 and tumor necrosis factor-alpha gene polymorphisms in Chinese children with Henoch-Schönlein purpura. World J Pediatr. 2016;12(1):49-54.

12. $\mathrm{Xu} \mathrm{H}, \mathrm{Pan} \mathrm{Y}, \mathrm{Li}$ W, et al. Association between IL17A and IL17F polymorphisms and risk of Henoch-Schönlein purpura in Chinese children. Rheumatol Int. 2016;36(6):829-835.

13. Hou HH, Huang YP, Liu L, et al. Association between CTLA-4 gene polymorphism and Henoch-Schönlein purpura in children [in Chinese]. Zhongguo Dang Dai Er Ke Za Zhi. 2017;19(3):296-302.

14. Wang BX, Mei H, Peng HM, et al. Association between platelet-activating factor acetylhydrolase gene polymorphisms and gastrointestinal bleeding in children with Henoch-Schönlein purpura [in Chinese]. Zhongguo Dang Dai Er Ke Za Zhi. 2017;19(4):385-388.

15. Wang A, Wang A, Xiao Y, Wang J, Xu E. Association of endothelial nitric oxide synthase gene polymorphism with susceptibility and nephritis development of Henoch-Schönlein purpura in Chinese Han children. Genet Test Mol Biomarkers. 2017;21(6):373-381.

16. Jiang J, Duan W, Shang $X$, et al. Inducible nitric oxide synthase gene polymorphisms are associated with a risk of nephritis in HenochSchönlein purpura children. Eur J Pediatr. 2017;176(8):1035-1045.

17. Xu H, Jiang G, Shen H, Li W, Mao J, Pan Y. Association of TLR4 gene polymorphisms with childhood Henoch-Schönlein purpura in a Chinese population. Rheumatol Int. 2017;37(11):1909-1915.

18. Pan $Y X, Y e Q$, Shao WX, et al. Relationship between immune parameters and organ involvement in children with Henoch-Schönlein purpura. PLoS One. 2014;9(12):e115261.

19. Ozen S, Pistorio A, lusan SM, et al; Paediatric Rheumatology International Trials Organisation (PRINTO). EULAR/PRINTO/PRES criteria for Henoch-Schönlein purpura, childhood polyarteritis nodosa, childhood Wegener granulomatosis and childhood Takayasu arteritis: Ankara 2008. Part II: Final classification criteria. Ann Rheum Dis. 2010;69(5):798-806.

20. Saulsbury FT. Henoch-Schönlein purpura in children. Report of 100 patients and review of the literature. Medicine (Baltimore). 1999; 78(6):395-409.

21. Saulsbury FT. Clinical update: Henoch-Schönlein purpura. Lancet. 2007;369(9566):976-978.

22. Ruperto N, Ozen S, Pistorio A, et al; Paediatric Rheumatology International Trials Organisation (PRINTO). EULAR/PRINTO/PRES criteria for Henoch-Schönlein purpura, childhood polyarteritis nodosa, childhood Wegener granulomatosis and childhood Takayasu arteritis: Ankara 2008. Part I: Overall methodology and clinical characterisation. Ann Rheum Dis. 2010;69(5):790-797.

23. Demetriou M, Granovsky M, Quaggin S, Dennis JW. Negative regulation of T-cell activation and autoimmunity by Mgat $5 \mathrm{~N}$-glycosylation. Nature. 2001;409(6821):733-739.

24. Brynedal B, Wojcik J, Esposito F, et al. MGAT5 alters the severity of multiple sclerosis. J Neuroimmunol. 2010;220(1-2):120-124.

25. Richter J, Benson V, Grobarova V, et al. CD161 receptor participates in both impairing NK cell cytotoxicity and the response to glycans and vimentin in patients with rheumatoid arthritis. Clin Immunol. 2010;136(1):139-147.

26. Morgan R, Gao G, Pawling J, Dennis JW, Demetriou M, Li B. N-acetylglucosaminyltransferase $\mathrm{V}$ (Mgat5)-mediated N-glycosylation negatively regulates Th1 cytokine production by T cells. J Immunol. 2004; 173(12):7200-7208.

27. Leech $M$, Metz $C$, Hall $P$, et al. Macrophage migration inhibitory factor in rheumatoid arthritis: Evidence of pro-inflammatory function and regulation by glucocorticoids. Arthritis Rheum. 1999;42(8):1601-1608.

28. Bloom BR, Bennett $B$. Mechanism of a reaction in vitro associated with delayed-type hypersensitivity. Science. 1966;153(3731):80-82.

29. Calandra T, Echtenacher B, Roy DL, et al. Protection from septic shock by neutralization of macrophage migration inhibitory factor. Nat Med. 2000;6(2):164-170.

30. Renner P, Roger T, Calandra T. Macrophage migration inhibitory factor: Gene polymorphisms and susceptibility to inflammatory diseases. Clin Infect Dis. 2005;41(Suppl 7):S513-519. 
31. Donn R, Alourfi Z, De Benedetti F, et al; British Paediatric Rheumatology Study Group. Mutation screening of the macrophage migration inhibitory factor gene: Positive association of a functional polymorphism of macrophage migration inhibitory factor with juvenile idiopathic arthritis. Arthritis Rheum. 2002;46(9):2402-2409.

32. Zheng WJ, Chen MG, Chen XY, et al. Renal expression of macrophage migration inhibitory factor in children with Henoch-Schönlein purpura nephritis [in Chinese]. Zhongguo Dang Dai Er Ke Za Zhi. 2010; 12(2):120-122.

33. Wang J, Li Y, Chen Y, et al. Urinary macrophage migration inhibitory factor as a noninvasive biomarker in pediatric Henoch-Schönlein purpura nephritis. J Clin Rheumatol. 2017;23(5):258-261.

34. Nalbantoglu S, Tabel Y, Mir S, Berdeli A. Lack of association between macrophage migration inhibitory factor gene promoter $(-173 \mathrm{G} / \mathrm{C})$ polymorphism and childhood Henoch-Schönlein purpura in Turkish patients. Cytokine. 2013;62(1):160-164.

35. Amoli MM, Martin J, Miranda-Filloy JA, Garcia-Porrua C, Ollier WE, Gonzalez-Gay MA. Lack of association between macrophage migration inhibitory factor gene $(-173 \mathrm{G} / \mathrm{C})$ polymorphism and cutaneous vasculitis. Clin Exp Rheumatol. 2006;24(5):576-579.
36. Morand EF, Leech M, Bernhagen J. MIF: A new cytokine link between rheumatoid arthritis and atherosclerosis. Nat Rev Drug Discov. 2006; 5(5):399-410.

37. Hoi AY, Iskander MN, Morand EF. Macrophage migration inhibitory factor: A therapeutic target across inflammatory diseases. Inflamm Allergy Drug Targets. 2007;6(3):183-190.

38. Calandra T, Bernhagen J, Metz CN, et al. MIF as a glucocorticoidinduced modulator of cytokine production. Nature. 1995;377(6544): 68-71.

39. Loureiro LR, Carrascal MA, Barbas A, et al. Challenges in antibody development against Tn and sialyl-Tn antigens. Biomolecules. 2015; 5(3):1783-1809.

40. Wang FF, Zhu LA, Zou YQ, et al. New insights into the role and mechanism of macrophage migration inhibitory factor in steroid-resistant patients with systemic lupus erythematosus. Arthritis Res Ther. 2012; 14(3):R103. 